\title{
Retardation of Corrected QT Dispersion Recovery, Heart Rate Recovery and Increase of QT Dispersion in Patients with Pseudoexfoliative Syndrome; Are They Predictors of Malignant Arrythmias?
}

\author{
Cosgun Ayhan 1*, Gunes Alper ${ }^{2}$, Oren Huseyin ${ }^{3}$ \\ ${ }^{1}$ Department of Cardiology, Cubuk State Hospital, Ankara, Turkey \\ ${ }^{2}$ Department of Ophtalmology, Cubuk State Hospital, Ankara, Turkey \\ ${ }^{3}$ Department of Cardiology, Sincan State Hospital, Ankara, Turkey \\ Email: *ayhancosgun@gmail.com, alpergunes@gmail.com, husoren@hotmail.com
}

How to cite this paper: Ayhan, C., Alper, G. and Huseyin, O. (2019) Retardation of Corrected QT Dispersion Recovery, Heart Rate Recovery and Increase of QT Dispersion in Patients with Pseudoexfoliative Syndrome; Are They Predictors of Malignant Arrythmias? World Journal of Cardiovascular Diseases, 9, 158-167.

https://doi.org/10.4236/wjcd.2019.93015

Received: January 26, 2019

Accepted: March 10, 2019

Published: March 13, 2019

Copyright $\odot 2019$ by author(s) and Scientific Research Publishing Inc. This work is licensed under the Creative Commons Attribution International License (CC BY 4.0).

http://creativecommons.org/licenses/by/4.0/

c) (i) Open Access

\begin{abstract}
Background: In pseudoexfoliative syndrome (PEX), extraocular accumulation is associated with cardiovascular morbidity and mortality. The aim of this study was to investigate the effect of exercise test on corrected QT dispersion (QTcd) and QTcd recovery in PEX. Patients and Methods: A total of 37 patients diagnosed as PEX were included in the study. The control group consisted of 38 patients. The Electrocardiographies (ECG) were taken. The patients were subjected to an exercise test. Results: In the study group, baseline, peak and third minute recovery QTcd values were $41.35 \pm 7.27 \mathrm{~ms}$, $65.19 \pm 7.83 \mathrm{~ms}$, and $53.61 \pm 6.45 \mathrm{~ms}$, respectively. In the control group, baseline, peak and third minute recovery QTcd values were $29.92 \pm 4.23 \mathrm{~ms}$, $38.31 \pm 4.22 \mathrm{~ms}$ and $28.76 \pm 3.09 \mathrm{~ms}$, respectively. There was statistically significant difference between the baseline and peak QTcd values of the study group and the control group $(\mathrm{t}=8.34224 / \mathrm{p}<0.00001, \mathrm{t}=18.55079 / \mathrm{p}<$ 0.00001 , respectively). In addition, while there was a statistically significant difference between the baseline QTcd and the third minute recovery QTcd in the study group, there was no statistically significant difference between the baseline QTcd value and the third minute recovery QTcd value in the control group as heart rate recovery $(\mathrm{t}=8.388558 / \mathrm{p}<0.00001, \mathrm{t}=$ $1.967315 / \mathrm{p}=0.056668$, respectively). Conclusion: All of them were found to have a higher risk in terms of malignant ventricular arrhythmia in the study group, both at rest and with effort, in a statistically significant way compared to the control group.
\end{abstract}




\section{Keywords}

Pseudoexfoliative Sydrome, Heart Rate Recovery, QTc Dispersion, Defective QTcd Recovery, Effort Test

\section{Introduction}

Pseudoexfoliative syndrome (PEX) is characterized by the accumulation of fibrillary extracellular material in some organs, in the eye and out of the eye. PEX can be easily identified by biomicroscopic examination with the presence of anterior segment changes characterized by pupillary border and white deposits on the anterior aspect of the lens [1]. Extra-ocular accumulation is associated with cardiovascular disease and mortality. It has been shown to be associated with PEX, myocardial infarction, ischemic heart disease, congestive heart failure, cardiomyopathy, aortic aneurysm and arterial hypertension [2] [3] [4]. The underlying cause of cardiovascular complications was impaired cardiovascular function [5].

It has been swowed that the rate of PEX in patients with coronary artery disease and the rate of coronary artery disease in patients with PEX had a greater incidence than healthy subjects [6]. In addition, PEX was closely associated with coronary artery disease in patients undergoing cataract surgery [7]. An increase in the chance of arrhythmia was also detected in PEX without coronary artery disease. This is thought to be due to changes in autonomic functions [8]. QT dispersion (QTd) is the value obtained by removing the shortest QT time from the longest QT duration in ECG and the value above $60 \mathrm{~ms}$ is known to be associated with lethal ventricular arrhythmias [9].

QTcd has been shown to be increased in PEX patients [10]. However, the effect of stress test on corrected QTdispersion (QTcd) in patients with PEX and QTcd recovery was not observed in this patient group.

In the literature on QTcd recovery, only one study has been conducted, and our study has been the second study using this term so far. Therefore, the purpose of our study was to investigate the effect of exercise test on QTcd and evalute QTcd recovery time in patients diagnosed with PEX in the ophthalmology outpatient clinic as compared with regard to heart rate recovery and the control group.

\section{Patients}

A total of 37 patients (19 male and 18 female) diagnosed with PEX by biomicroscopic examination, with observation of the substance called gray white fibrogranular pseudoexfoliation material at the edge of the lens anterior capsule and/or pupil, were enrolled in the study. This group was determined as the study group. The mean age of the study group was $64.7 \pm 9.6$ years. The control group 
consisted of similar age and gender group patients who did not have any pathology in their ophthalmologic examinations and who did not have any disease. A total of 38 patients were included in the control group. 18 of the patients were female and 20 were male. The mean age of the control group was $63.8+/-8.3$ years. The exclusion criteria were, having systemic diseases such as hypertension and diabetes mellitus, having coronary artery disease, having heart failure or having a major valve disease in routine transthoracic echocardiography, electrolyte disorder in routine blood examination, anemia, having impaired thyroid function test, smoking, having alchol use and taking any drugs which can effect the heart, having blood pressure above $140 / 90 \mathrm{~mm} \mathrm{Hg}$ during physical examination, being too fond of exercise test or having orthopedic disorder and being not to reach $88 \%$ of target pulse.

\section{Methods}

After PEX was diagnosed by biomicroscopy, routine blood tests, hemogram and thyroid function tests were performed. Patients without any electrolyte disorder, anemia or thyroid, renal and hepatic dysfunction were included in the study and those with any systemic disease such as electrolyte disorder, hypertension or diabetes mellitus, anemia, thyroid, liver and kidney dysfunction were excluded from the study. The study group was then subjected to transthoracic echocardiography by a cardiologist. Patients with left ventricular ejection fraction below $60 \%$ and significant valvular disease were excluded from the study. After transthoracic echocardiography, resting ECG recordings were taken at a rate of $50 \mathrm{~mm} / \mathrm{sec}$ in the semi-trandelenburg position after a 5 minute rest period. The patients were then subjected to stress test according to Bruce protocol [11]. Those with arterial tension above 140/90 $\mathrm{mm} \mathrm{hg}$ were excluded from the study.The test was terminated when the patients reached $88 \%$ of the target pulse. Recordings were taken at a speed of $50 \mathrm{~mm} / \mathrm{sec}$. The control group also underwent routine physical examination, blood test and transthoracic echocardiography, then atfer the ECG recordings were taken at $50 \mathrm{~mm} / \mathrm{sec}$ speed, they were taken to exercise test according to Bruce protocol. Those whose arterial tension was above $140 / 90 \mathrm{~mm}$ hg were excluded from the study. The test was terminated when the patients reached $88 \%$ of the target rate. Calculations are made with digital ruler by a cardiologist. While QT duration measured from the beginning of the Q wave in the QRS to the end of the T wave, QT dispersion was obtained by removing the shortest QT time from the longest QT time. QT and QTd were obtained by multiplying each measured small square by $40 \mathrm{msec}$. The calculations were performed on all leads in the records and at least three consecutive QRS waves. Patients who failed to compute in a lead and in 3 consecutive QRS waves were excluded from the study or the record was repeated. Then, corrected QT (QTc) and QTcd was calculated as using Framingham Correction [12]. All of these evaluations were performed for both the study group and the control group. 


\section{Statistical Analysis}

SPSS software [Version X; IBM, Armonk, NY, USA] 20.0 was used for the collection and analysis of the data. Measurements were expressed as mean values with \pm Standard deviation. Analyzes were performed using t-test. For all tests $\mathrm{p}$ $<0.05$, the differences are statistically significance.

\section{Results}

There was no statistically significant difference between sociodemographic and clinical characteristics of the participants of study and control group (Table 1).

There was no statistically significant difference between baseline and peak exercise QTc values in the study group and the control group, respectively. However, when QTcd values were taken into consideration, there was a statistically significant difference between basal and peak QTcd values in the control group and study group (Table 2).

There was a statistically significant difference between basal QTc and third minute recovery QTc in study group. There was a statistically significant difference between basal and third minute recovery QTcd in study group (Figure 1). There was a statistically significant difference between basal and third minute recovery heart rate in study group (Table 3 ).

There was statistically no significant difference between basal and third minute recovery QTc values, basal QTcd and third minute QTcd (Figure 1), basal heart rate and third minute heart rate in the control group (Table 4).

\section{Discussion}

In our study, two important results emerged. The first one is that the resting and peak exercise QTcd values were significantly higher in the study group

Table 1. Sociodemographic and clinical characteristics of the participants.

\begin{tabular}{cccc}
\hline Variables & Study group $(\mathbf{n}=\mathbf{3 7})$ & Control group $(\mathbf{n}=\mathbf{3 8})$ & p value \\
\hline Sex & 19 male $(\% 51.35)$, & 20 male $(\% 52.63)$, & $\mathrm{p}>0.05$ \\
Age & 18 female $(\% 48.65)$ & 18 female $(\% 47.37)$ & $\mathrm{p}>0.05$ \\
Blood pressure & $125.35 \pm 13.55 \mathrm{~mm} \mathrm{Hg}$ & $123.44 \pm 12.84 \mathrm{~mm} \mathrm{Hg}$ & $\mathrm{p}>0.05$ \\
Basal Heart Rate & $71 \pm 12.48$ & $74.73 \pm 13.58$ & $\mathrm{p}>0.05$ \\
BMI & $27.33 \pm 5.7$ & $28.69 \pm 4.9$ & $\mathrm{p}>0.05$ \\
LV mass, gr & $175.48 \pm 33.58$ & $177.28 \pm 28.73$ & $\mathrm{p}>0.05$ \\
Total cholesterol, & $198.55 \pm 43.78$ & $202.36 \pm 36.73$ & $\mathrm{p}>0.05$ \\
mg/dlt & $137.38 \pm 46.63$ & $144.93 \pm 38.47$ & $\mathrm{p}>0.05$ \\
LDL, mg/dlt & $157.55 \pm 56.73$ & $149.82 \pm 63.81$ & $\mathrm{p}>0.05$ \\
Triglycerides, & $14.63 \pm 2.2$ & $13.76 \pm 2.6$ & $\mathrm{p}>0.05$ \\
mg/dlt & $9.4 \pm 0.7$ & $9.1 \pm 0.8$ & $\mathrm{p}>0.05$ \\
Hemoglobin, gr/dlt & $142.58 \pm 5.77$ & $139.47 \pm 4.68$ & $\mathrm{p}>0.05$ \\
Calcium, mg/dlt & years & \\
Sodium, mEq/L & & &
\end{tabular}

Abr; BMI: Body Mass Index, LV: Left Ventricle, LDL: Low Density Cholesterol. 
Table 2. The QTc and QTcd values obtained from ECG and treadmill exercise test of the study and control group.

\begin{tabular}{cccc}
\hline Variables & Study group & Control group & $\mathrm{t}$ and $\mathrm{p}$ value \\
\hline basal QTc, ms & $397.16 \pm 16.77$ & $399.79 \pm 17.07$ & $\mathrm{t}=0.67196$ and $\mathrm{p}=0.251867$ \\
peak exercise QTc, & $417.84 \pm 14.61$ & $417.37 \pm 15.33$ & $\mathrm{t}=0.13568$ and $\mathrm{p}=0.446226$ \\
basal QTcd, ms & $41.35 \pm 7.27$ & $29.92 \pm 4.23$ & $\mathrm{t}=8.34224$ and $\mathrm{p}<0.00001$ \\
peak exercise QTcd & $65.19 \pm 7.83$ & $38.31 \pm 4.22$ & $\mathrm{t}=18.55079$ and $\mathrm{p}<0.00001$ \\
\hline
\end{tabular}

QTc: Corrected QT, QTcd: Corrected QT dispersion.

Table 3. The QTc and QTcd HR values obtained from treadmill exercise test of study group.

\begin{tabular}{ccc}
\hline Variable 1 & Variable 2 & t and p value \\
\hline basal QTc, 397.16 $\pm 16.77 \mathrm{~ms}$ & thr. min QTc, 406.40 $\pm 12.78 \mathrm{~ms}$ & $\mathrm{t}=7.02, \mathrm{p}<0.00001$ \\
basal QTcd, $41.35 \pm 7.27 \mathrm{~ms}$ & third min QTcd, 53.61 $\pm 6.45 \mathrm{~ms}$ & $\mathrm{t}=8.38, \mathrm{p}<0.00001$ \\
basal HR, 83.45 $\pm 10.43 \mathrm{beat} / \mathrm{min}$ & third min HR, 91.23 $\pm 7.73 \mathrm{~b} / \mathrm{mn}$ & $\mathrm{t}=3.98, \mathrm{p}=0.000157$ \\
\hline
\end{tabular}

QTc: Corrected QT, QTcd: Corrected QT dispersion.

Table 4. The QTc and QTcd HR values obtained from treadmill exercise test of control group.

\begin{tabular}{ccc}
\hline Variable 1 & Variable 2 & $\mathrm{t}$ and $\mathrm{p}$ value \\
\hline basal QTc, 399.79 $\pm 17.07 \mathrm{~ms}$ & trd.min QTc, 399.05 $\pm 16.72 \mathrm{~ms}$ & $\mathrm{t}=1.6862, \mathrm{p}=0.11402$ \\
Basal QTcd, 29.92 $\pm 4.23 \mathrm{~ms}$ & trd.min QTcd, 28.76 $\pm 3.09 \mathrm{~ms}$ & $\mathrm{t}=1.9673, \mathrm{p}=0.056668$ \\
basal HR, 80.60 $\pm 10.81 \mathrm{beat} / \mathrm{min}$ & third min HR, 81.34 $\pm 11.37 \mathrm{~b} / \mathrm{mn}$ & $\mathrm{t}=1.24249, \mathrm{p}=0.22187$
\end{tabular}

QTc: Corrected QT, QTcd: Corrected QT dispersion, HR: Heart Rate.

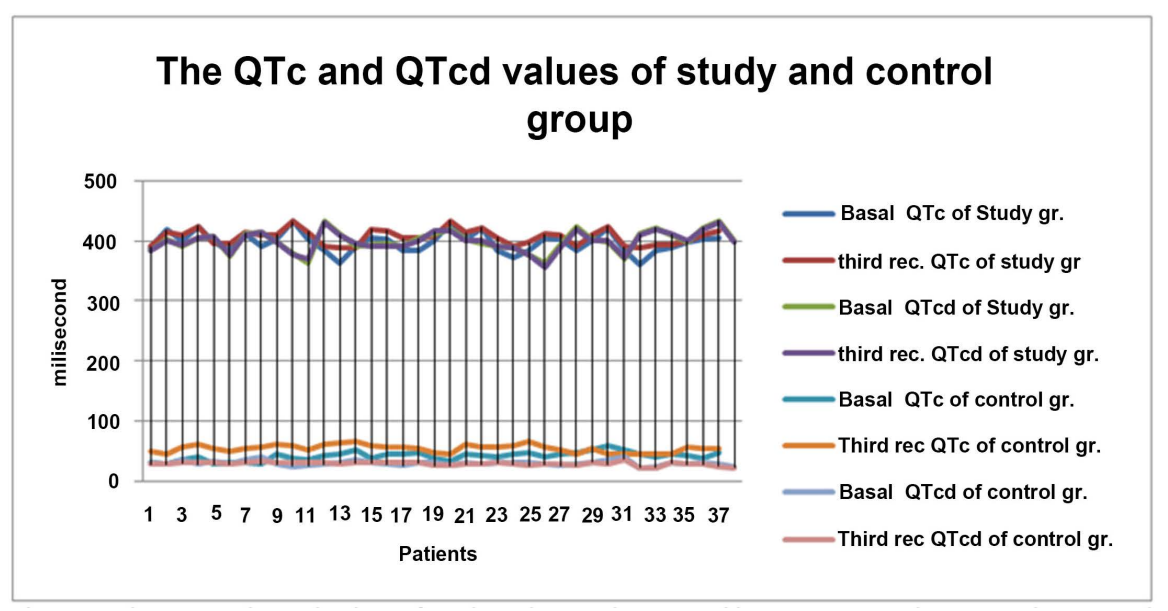

Figure 1. The QTc and QTcd values of Study and Control Group QTc: Corrected QT, QTcd: Corrected QT dispersion.

compared to the control group. The second important result was that there was a statistically significant difference between third minute recovery QTcd values 
and baseline QTcd values in the study group. In the control group, the difference between third minute recovery QTcd and baseline QTcd was statistically insignificant in the third minute. This means that there was a defective QTcd recovery in the third minute after the exercise test in the study group compared to the control group.

Although the relationship between PEX and many organ diseases is shown closely, the most important of these is its association with cardiovascular events [13]. Cardiovascular disease most commonly associated with PEX is undoubtedly coronary artery disease [5]. In one study, the incidence of coronary artery disease in patients with PEX and the incidence of PEX in patients with coronary artery disease were found to be increased [6]. It has been also advocated that coronary artery disease has increased in PEX patients over the age of 50 years [7]. Other major diseases associated with PEX; hypertension and angina pectoris [14], serbrovascular events [15], alzheimer disease [16], aortic aneurysm [17] and peripheral artery disease [18].

Those who investigated the relationship between PEX and cardiovascular disease have focused on many mechanisms. The first is the hemocystein level, which is found to be increased in PEX patients compared to non-PEX patients [19] [20]. Although there was an increase in homocysteine levels, vitamin B12, folic acid and B6 levels were decreased in PEX patients [21]. However, in one study, the association between PEX and coronary artery disease, hypertension, cerebrovascular events, diabetes mellitus and arrhythmias was investigated and it was suggested that only the prevalence of arrhythmia was high in PEX patients [22]. In addition to all these, although there was a minority, it has been claimed that there was no association between PEX and cardiovascular disease [23].

The mechanisms underlying the arrhythmias associated with PEX have been investigated and it was suggested that most prominent of these was the defective autonomic system [24]. To prove this, the carotid artery baroreseptor reflex was investigated and a decreased and impaired vagal activity was found in PEX patients [25]. In addition, the same group carried out another study to support these findings and reported decreased cardio-vagal regulation [26]. The most emphasized parameter in Vagal autonomic system failure was Hearth Rate Recovery. A defective heart rate recovery is associated with increased cardiovascular mortality [27]. However, QTcd recovery has never been studied. In our study, we found that tehere was a defective heart rate rocovery in the PEX group, compared with the control group. Also the defective QTcd recovery has been found in the study group, as defective as heart rate recovery. This means that QTcd recovery can indirectly indicate vagal activity, such as heart rate recovery.

Disruption of ventricular repolarization may lead to malignant ventricular arrhythmias in patients. Coronary artery disease and long QT duration are the most important reasons for ventricular arrhythmias [28]. An increased QT duration and QTcd duration were associated with sudden cardiac death [29]. The QTc value was greater than $450 \mathrm{~ms}$ and the QTcd value above $60 \mathrm{~ms}$ was associated with malignant ventricular arrhythmias and sudden cardiac death 
[30] [31]. In addition, QTcd levels were found to be significantly higher in PEX patients than in the control group, but the relationship with the stress test was not investigated [10]. In addition, only one study on impaired QTcd recovery was reported in the literature and it was found to be an independent predictor of mortality at 6 months of follow-up [32].

In our study, we found that the baseline and peak exercise QTcd values were significantly higher in the study group than the control group. This suggests that PEX patients may have a greater risk of malignant ventricular arrhythmias, both at rest and with exertion, which may be more common than normal people.In addition, we found that PEX patients were defective QTcd recovery as defective as heart rate recovery, compared to the control group.

\section{Conclusion}

In conclusion, PEX patients have a risk of malignant ventricular arrhythmia, both at rest and with effort. Furthermore, delayed QTcd recovery times showing the indirect presence of vagal activity were defective. This finding also supports the increased risk of arrhythmias.

\section{Study Limitations}

In our study, the number of cases is little. Healthier data can be made with larger case studies. And also, we calculated QTc and QTcd values as manually by digital caliper. In addition, long-term studies should be made in order to observe the effect of medical therapy on the QTc, QTcd and ventricular arrythmias induced by the exercise test in patients with PEX. More comprehensive and long-term follow-up studies should be performed to obtain healthier data.

\section{Ethical Approval}

All procedures performed in studies involving human participants were in accordance with the ethical standards of the institutional and/or national research committee and with the 1964 Helsinki declaration and its later amendments or comparable ethical standards. There was no ethics committee in our hospital. The autors state that the manuscript has been read and approved by all the authors, that the requirements for authorship as stated earlier in this document have been met, and that each author believes that the manuscript represents honest work. Dr Alper Gunes provided and examined the PEX patients and Dr. Ayhan Cosgun and Dr Huseyin Oren made cardiac examinations, and together they wrote the article.

\section{Conflicts of Interest}

The authors declare that they have no conflict of interest. There is no source(s) of support in the form of grants, equipment, drugs. All expenses are paid by the authors. 


\section{References}

[1] Wang, W., He, M., Zhou, M. and Zhang, X. (2014) Ocular Pseudo Exfoliation Syndrome and Vascular Disease: A Systematic Review and Meta Analysis. PLoS ONE, 9, e92767. https://doi.org/10.1371/journal.pone.0092767

[2] Sekeroglu, M.A., Bozkurt, B., Irkec, M., et al. (2008) Systemic Associations and Prevalence of Exfoliation Syndrome in Patients Scheduled for Cataract Surgery. European Journal of Ophthalmology, 18, 551-555. https://doi.org/10.1177/112067210801800408

[3] Gone, K.A., Gone, T. and Gumus, B. (2012) Reply: Pseudoexfoliation Syndrome and Cardiovascular Disease: Studies Must Control for All Cardiovascular Risk Factors. Eye, 27, 1329. https://doi.org/10.1038/eye.2013.185

[4] Schumacher, S., Schlötzer-Schrehardt, U., Martus, P., et al. (2001) Pseudoexfoliation Syndrome and Aneurysms of the Abdominal Aorta. The Lancet, 357, 359-360. https://doi.org/10.1016/S0140-6736(00)03645-X

[5] Mitchell, P., Wang, J.J. and Smith, W. (1997) Association of Pseudoexfoliation Syndrome with İncreased Vascular Risk. American Journal of Ophthalmology, 124, 685-687. https://doi.org/10.1016/S0002-9394(14)70908-0

[6] Citirik, M., Acaroglu, G., Batman, C., Yildiran, L. and Zilelioglu, O. (2007) A Possible Link between the Pseudo Exfoliation Syndrome and Coronary Artery Disease. Eye (London), 21, 11-15. https://doi.org/10.1038/sj.eye.6702177

[7] Andrikopoulos, G.K., Mela, E.K., Georgakopoulos, C.D., Papadopoulos, G.E., Damelou, A.N., Alexopoulos, D.K., et al. (2009) Pseudoexfoliation Syndrome Prevalence in Greek Patients with Cataract and İts Association to Glaucoma and Coronary Artery Disease. Eye (London), 23, 442-447. https://doi.org/10.1038/sj.eye.6702992

[8] Brajkovic, J., Surac, I.K., Ercegovic, A., Miletic-Juric, A., Susic, N. and Buric, Z. (2007) Ocular Pseudo Exfoliation Syndrome and Internal Systemic Diseases. Acta Clinica Croatica, 46, 57-61.

[9] Moreno, F.L., Villanueva, M.T., Karagounis, L.A. and Anderson, J.L. (1994) Reduction in QT Interval Dispersion by Successful Thrombolytic Therapy in Acute Myocardial İnfarction. Circulation, 90, 94-100. https://doi.org/10.1161/01.CIR.90.1.94

[10] Demir, A.K., Demir, S., Arisoy, A., et al. (2017) The Association of Pseudoexfoliation Syndrome with Ventricular Repolarization Dynamics. Asian Journal of Medicine and Health, 5, 1-7.

[11] Will, P.M. and Walter, J.D. (1999) Exercise Testing: İmproving Performance with a Ramped Bruce Protocol. American Heart Journal, 138, 1033-1037. https://doi.org/10.1016/S0002-8703(99)70067-0

[12] Vandenberg, B., Vandael, E., Robyns, T., Vandenberge, J., et al. (2016) Framingham Correction, Which QT Correction Formulae to Use for QT Monitoring? Journal of the American Heart Association, 5, e003264.

[13] Naumann, G.O., Schlötzer-Schrehardt, U. and Küchle, M. (1998) Pseudoexfoliation Syndrome for the Comprehensive Ophthalmologist. Intraocular and Systemic Manifestations. Ophthalmology, 105, 951-968. https://doi.org/10.1016/S0161-6420(98)96020-1

[14] Miyazaki, M., Kubota, T., Kubo, M., Kiyohara, Y., Iida, M., Nose, Y., et al. (2005) The Prevalence of Pseudoexfoliation Syndrome in a Japanese Population: The Hisayama Study. Journal of Glaucoma, 14, 482-484. https://doi.org/10.1097/01.ijg.0000185436.15675.b3 
[15] Ritland, J.S., Egge, K., Lydersen, S., Juul, R. and Semb, S.O. (2004) Exfoliative Glaucoma and Primary Open-Angle Glaucoma: Associations with Death Causes and Comorbidity. Acta Ophthalmologica Scandinavica, 82, 401-404. https://doi.org/10.1111/j.1395-3907.2004.00297.x

[16] Linnér, E., Popovic, V., Gottfries, C.G., Jonsson, M., Sjögren, M. and Wallin, A. (2001) The Exfoliation Syndrome in Cognitive Impairment of Cerebrovascular or Alzheimer's Type. Acta Ophthalmologica Scandinavica, 79, 283-285. https://doi.org/10.1034/j.1600-0420.2001.790314.x

[17] Djordjevic-Jocic, J., Jovanovic, P., Bozic, M., Tasic, A. and Rancic, Z. (2012) Prevalence and Early Detection of Abdominal Aortic Aneurysm in Pseudoexfoliation syndrome and Pseudoexfoliation Glaucoma. Current Eye Research, 37, 617-623. https://doi.org/10.3109/02713683.2012.665120

[18] Praveen, M.R., Shah, S.K., Vasavada, A.R., Diwan, R.P., Shah, S.M., Zumkhawala, B.R., et al. (2011) Pseudoexfoliation as a Risk Factor for Peripheral Vascular Disease: A Case-Control Study. Eye, 25, 174-179. https://doi.org/10.1038/eye.2010.175

[19] Bleich, S., Roedl, J., Von Ahsen, N., Schlötzer-Schrehardt, U., Reulbach, U., Beck, G., et al. (2004) Elevated Homocysteine Levels in Aqueous Humor of Patients with Pseudoexfoliation Glaucoma. American Journal of Ophthalmology, 138, 162-164. https://doi.org/10.1016/j.ajo.2004.02.027

[20] Roedl, J.B., Bleich, S., Reulbach, U., Rejdak, R., Kornhuber, J., Kruse, F.E., et al. (2007) Homocysteine in Tear Fluid of Patients with Pseudoexfoliation Glaucoma. Journal of Glaucoma, 16, 234-239. https://doi.org/10.1097/IJG.0b013e31802d6942

[21] Roedl, J.B., Bleich, S., Reulbach, U., Rejdak, R., Naumann, G.O., Kruse, F.E., et al. (2007) Vitamin Deficiency and Hyperhomocysteinemia in Pseudoexfoliation Glaucoma. Journal of Neural Transmission, 114, 571-575. https://doi.org/10.1007/s00702-006-0598-Z

[22] Ercegoviæ, A., Brajkoviæ, J., Suraæ, I.K. and Haluzan, M.B. (2012) Prevalence, Distribution and Types of Corneal Astigmatism in Cataract Surgery Patients in Sibenik County. Acta Clinica Croatica, 51, 275-278.

[23] Emiroglu, M.Y., Coskun, E., Karapinar, H., Capkýn, M., Kaya, Z., Kaya, H., et al. (2010) Is Pseudoexfoliation Syndrome Associated with Coronary Artery Disease? North American Journal of Medical Sciences, 2, 487-490. https://doi.org/10.4297/najms.2010.2487

[24] Hollo, G., Lakatos, P. and Farkas, K. (1998) Cold Pressor Test and Plasma Endothelin-1 Concentration in Primary Open-Angle and Capsular Glaucoma. Journal of Glaucoma, 7, 105-110.

[25] Visontai, Z., Merisch, B., Kollai, M. and Hollo, G. (2006) Increase in Carotid Artery Stiffness and Decrease of Baroreflex Sensitivity in Exfoliation Syndrome and Glaucoma. British Journal of Ophthalmology, 90, 563-567.

https://doi.org/10.1136/bjo.2005.087908

[26] Visontai, Z., Horváth, T., Kollai, M. and Holló, G. (2008) Decreased Cardiovagal Regulation in Exfoliation Syndrome. Journal of Glaucoma, 17, 133-138. https://doi.org/10.1097/IJG.0b013e3181379d67

[27] Peçanha, T., Silva-Júnior, N.D. and Forjaz, C.L. (2014) Heart Rate Recovery: Autonomic Determinants, Methods of Assessment and Association with Mortality and Cardiovascular Diseases. Clinical Physiology and Functional Imaging, 34, 327-339. https://doi.org/10.1111/cpf.12102

[28] Zipes, D.P. and Wellens, H.J. (1998) Sudden Cardiac Death. Circulation, 98, 2334-2351. https://doi.org/10.1161/01.CIR.98.21.2334 
[29] De Bruyne, M.C., Hoes, A.W., Kors, J.A., Hofman, A., vanBemmel, J.H. and Grobbee, D.E. (1999) Prolonged QT Interval Predicts Cardiac and All-Cause Mortality in the Elderly. The Rotterdam Study. European Heart Journal, 20, 278-284. https://doi.org/10.1053/euhj.1998.1276

[30] Viskin, S. (1999) Long QT Syndromes and Torsade de Pointes. The Lancet, 354, 1625-1633. https://doi.org/10.1016/S0140-6736(99)02107-8

[31] Yamaguchi, M., Shimizu, M., Ino, H., Terai, H., Uchiyama, K., Oe, K., et al. (2003) T Wave Peak-to-End Interval and QT Dispersion in Acquired Long QT Syndrome: A New İndex for Arrhythmogenicity. Clinical Science, 105, 671-676. https://doi.org/10.1042/CS20030010

[32] Nuis, R.J., Turgut, G., van der Boon, R.M., et al. (2015) Defective Recovery of QT Dispersion Following Transcatheter Aortic Valve Implantation: Frequency, Predictors and Prognosis. Journal of Geriatric Cardiology, 12, 482-488. 